\title{
Noise Reduction in Optical Communication System \\ Gunjan
}

University School of Information, Communication \& Technology Guru Gobind Singh Indraprastha University, New Delhi-110078, India

Email: sharma.gini@gmail.com

Ghanendra Kumar*

Department of Electronics and Communication Engineering,National Institute of Technology, Delhi-110040, India

*corresponding author Email: gkumar3c@gmail.com

Chakresh Kumar

University School of Information, Communication \& Technology Guru Gobind Singh Indraprastha University, New Delhi-110078, India

Email:chakreshk@gmail.com

\section{ABSTRACT}

The aim of this paper to find best suited filter for given photo detector i.e APD and PIN for the suppression of noise in the proposed optical system. This paper also analysis the effect of SOA for noise reduction in the optical communication system. It is concluded that APD photodiode gives a better Performance in given optical system in comparison with PIN photodiode. Low pass Bessel filter shows the best performance among the other filters at $1310 \mathrm{~nm}$ wavelength. Bessel filter provides constant group delay. Eye opening height is more in Bessel filter. It gives minimum Bit error rate in received signal. Semiconductor optical amplifier reduces the noise in optical system. It improves the quality of received signal. It provides lower bit error rate upto $20 \mathrm{~km}$ optical fiber.

Keywords - BER, Eye-diagram, Filter, Optical source, Photo diodes.

\section{INTRODUCTION}

W hen the optical source emits light due to long distance transmission of light there is occurrence of noise. At the receiving section this noise effects the performance of received signal like BER, Q factor etc. This proposed system design to reduce noise in the received signal. First we try to check which filter well situated for better performance of given system and which photo detector work smoothly for better operation of system with lower bit error rate and high quality factor[1-4].

In the last section of this paper it is also discussed about the semiconductor optical amplifier responsible for noise reduction for the improvement of system functionality [5-7].

\subsection{Optical Source}

In this system Continuous-wave laser is used. It is continuously pumped and emits light. The operating frequency is $1550 \mathrm{~nm}$ and $1310 \mathrm{~nm}$ respectively. It operates at $10 \mathrm{~mW}$ power.

\subsection{Mach-Zehnder Modulator}

Mach Zehnder works on the principle of electro- electrooptic effect. By applying a external voltage optical path length is changed.

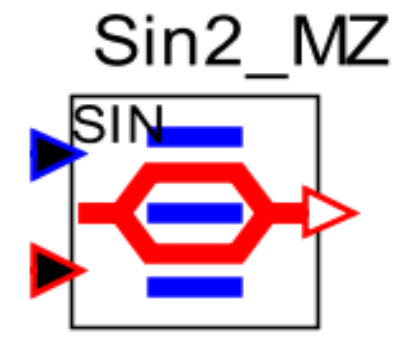

Fig. 1. Mach-Zehnder modulator

A Mach-Zehnder modulator controls the amplitude of an optical wave. The input waveguide split into two waveguide arms. Voltage is applied across one arm, it gives rise to phase shift when light wave passes through that arm. These two arms recombined the phase difference between two arms waves responsible for the amplitude modulation.

Delaying signal of one arm with respect to another arm. This delay is achieved by changing refractive index of one arm. This refractive index change is proportional to external applied voltage across one arm. The output is a result of interference between two signal combinations. When phase of light is in phase there is constructive interference. If phase in each arm differ by $\pi$ destructive interference occurs. Hence this modulator modulated the intensity of light. 


\subsection{Optical fiber}

In this proposed system single mode fiber is used. It provides high data rate and efficient long " transmission of signal. It is not suffered from modal dispersion. In this set up I am uing $10 \mathrm{~km}$ single mode fiber.

\subsection{Photo Detectors}

Photo detectors converts optical energy into electrical energy. Photo detector performance depends upon the responsivity of that detector. The responsivity decides generated amount of photocurrent for the amount of optical power incident on the photo detector.

\subsubsection{APD Photo Diode}

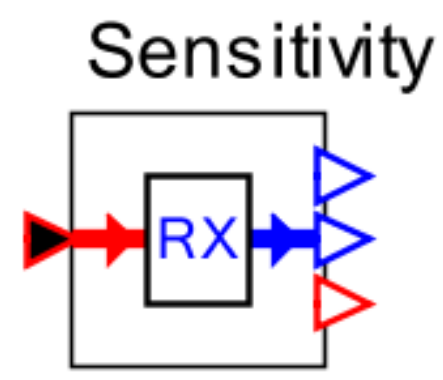

Fig. 2. APD Photo Diode

Avalanche photodiodes (APDs) are specially designed for reverse breakdown region. This diode has High level of sensitivity. It provides higher gain due to multiplication effect.

\subsubsection{PIN Photo Diode}

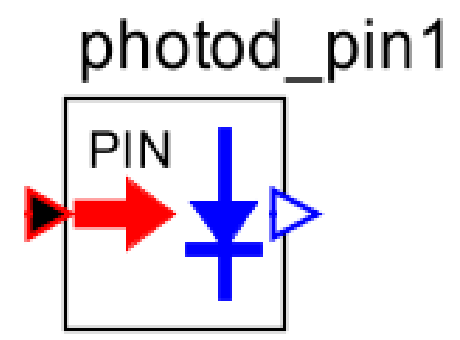

Fig. 3. PIN Photo Diode

A Pin diode contains intrinsic semiconductor layer between the p-type semiconductor and n-type semiconductor layer. Intrinsic layer is made by pure semiconductor material. The p-type and n-type layer is heavily doped.

\subsection{Filters}

\subsubsection{Butterworth Filter}

This filter has flat frequency response in the pass band.

\subsubsection{Chebyshev Filter}

This filter will allow ripples in the pass band amplitude response. It has steeper roll-off near cut off frequency compared with the butterworth filter. This filter has Poorer group delay.

\subsubsection{Bessel Filter}

The Bessel filter provides constant group delay.

\section{SIMULATION OF FILTERS AT DIFFERENT OPTICAL SOURCE WAVELENTHS AND PHOTODIODES}

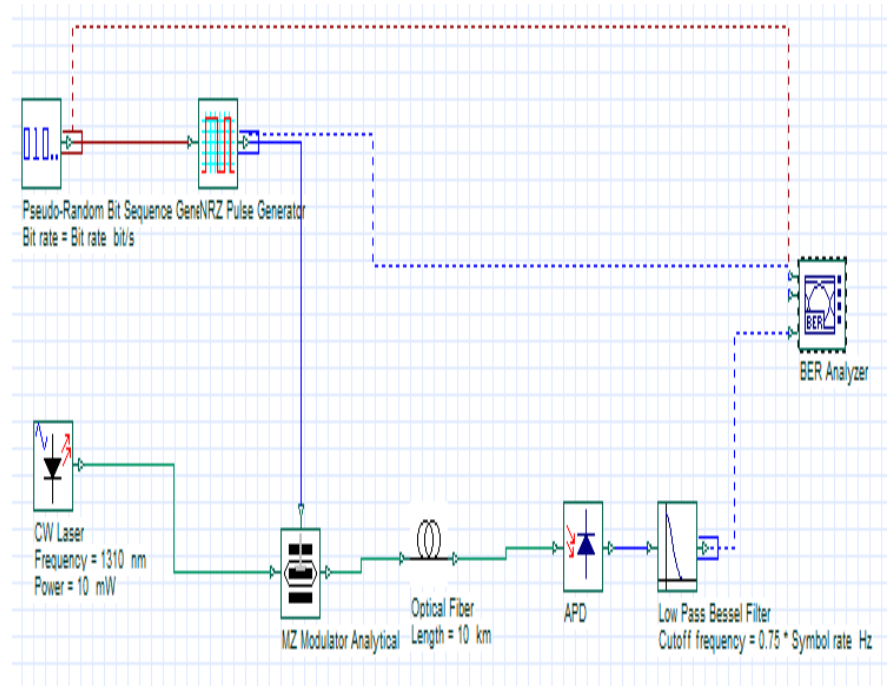

Fig. 4. Experimental Set up of Proposed Optical Communication System

\section{1 Optical System Design}

Optical system has been designed for the improvement in the noise and for simulation Optisystem 16.0. The proposed optical system given in fig.4. In the proposed system, first is optical transmitter, then a optical fiber link and at the last optical detector. In transmitter first section continuous wave (CW) laser of wavelength $1310 \mathrm{~nm}$ or $1550 \mathrm{~nm}$ is used as an optical source at $10 \mathrm{mw}$ power. The Pseudo random bit sequence which generates the sequence of ones and zeros in random fashion. The PRBS output is fed to NRZ pulse generator. Signal coded by NRZ scheme is generated by NRZ pulse generator. One terminal of MZ modulator connected with NRZ generator output and another terminal Mach-Zehnder modulator connected with output of CW laser source.

After modulation through $\mathrm{MZ}$ modulator signal is transmitted through a channel made by optical fiber of length $10 \mathrm{~km}$. The optical detector may be an APD or PIN photodiodes which detects the signal.

The photo detector converts the light signal into electrical signal. At the receiver end there is a low pass filter. This signal after filtration fed to BER analyzer. This analyzer is used to analysis the Q factor, BER and eye diagram of received signal. 


\section{SIMULATION EFFECT OF PERFORMANCE OF PROPOSED \\ AND ANALYSIS OF SOA ON THE}

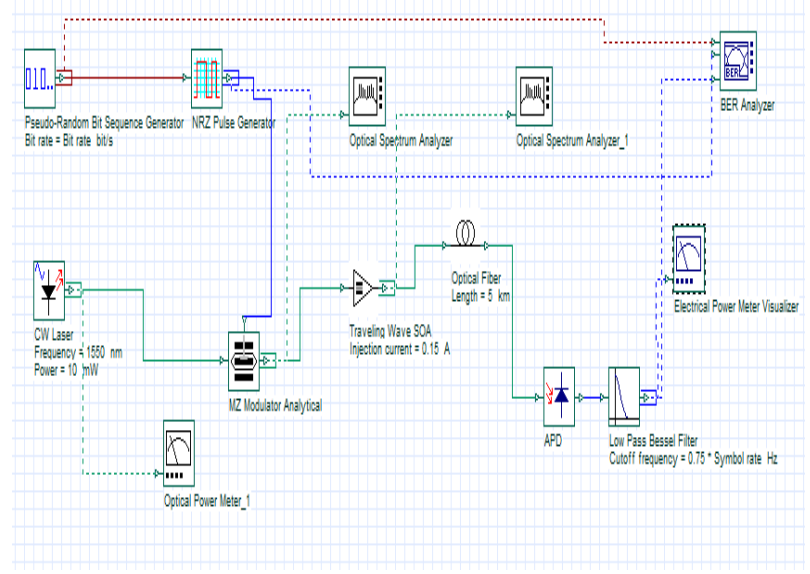

Fig. 5. Experimental set of upgraded optical system with SOA.

In this set up we insert semiconductor optical amplifier for the better performance of optical system. Input power of optical source is varied to test the operation of SOA. At different input power it provides different values of signal and noise power. Some bits changed due to noise in optical communication channel bit error rate gives us idea bits in error in the received signal data. SOA gives low BER hence it suppressed the noise. It provides high signal to noise ratio.

昌

Min. log of BER (Length (km)) 总 Min. log of BER (Lenght (km))

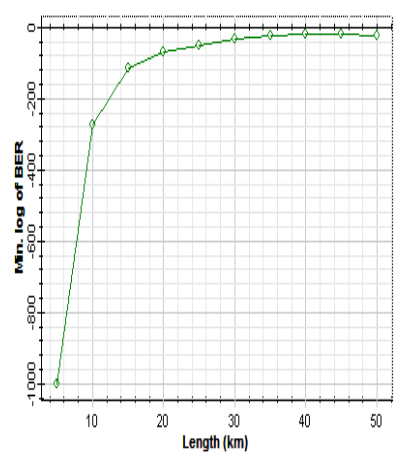

(a) Without SOA

Fig. 6. BER varies according to length of optical fiber

\section{RESULTS AND DISCUSSION}

In this proposed system, the performance of optical system having $10 \mathrm{~km}$ optical fiber has been analyzed on the basis of operation of various filters with combination of various types of photo detectors and at different wavelengths. The simulation is done on optisystem 16.0. After simulation I get the comparative result of the performance of photodiodes such as avalanche photodiodes (APD) and
PIN photodiodes on the basis of their quality factor at two different wave lengths $1550 \mathrm{~nm}$ and $1310 \mathrm{~nm}$ respectively.

Table 1: Quality Factor Obtained by using Filters and different Wavelengths

\begin{tabular}{|c|c|c|c|c|}
\hline $\begin{array}{c}\text { Photo } \\
\text { Detector } \\
\text { Type }\end{array}$ & $\begin{array}{c}\lambda \\
(\mathbf{n m})\end{array}$ & $\begin{array}{c}\text { Low Pass } \\
\text { Chebyshev } \\
\text { Filter }\end{array}$ & $\begin{array}{c}\text { Low Pass } \\
\text { Butterworth } \\
\text { Filter }\end{array}$ & $\begin{array}{c}\text { Low Pass } \\
\text { Bessel } \\
\text { filter }\end{array}$ \\
\hline PIN & 1550 & 20.46 & 23.01 & 36.40 \\
\hline PIN & 1310 & 37 & 28 & 59.38 \\
\hline APD & 1550 & 20.50 & 23.38 & 36.64 \\
\hline APD & 1310 & 37.24 & 28.10 & 59.94 \\
& & & & \\
\hline
\end{tabular}

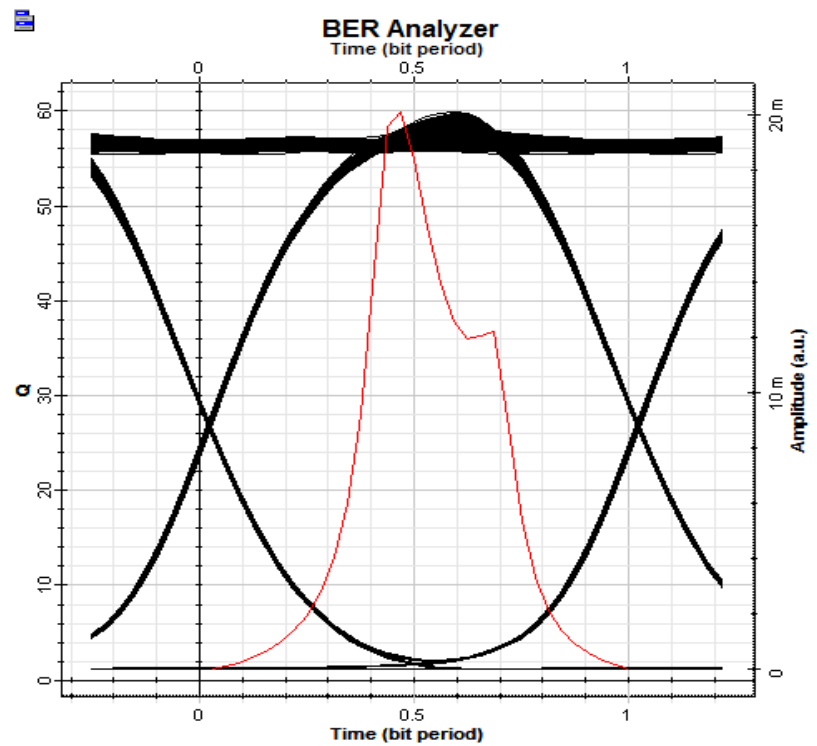

Fig. 7. Q Factor of Low pass Bessel filter

Fig. 7. shows the eye diagram of a optical system having wavelength $1310 \mathrm{~nm}$. The modulation coding is NRZ with photo detector APD is used. In this eye diagram we get result that the $\mathrm{Q}$ factor in this system is highest for low pass bessel filter is 59.94 . 


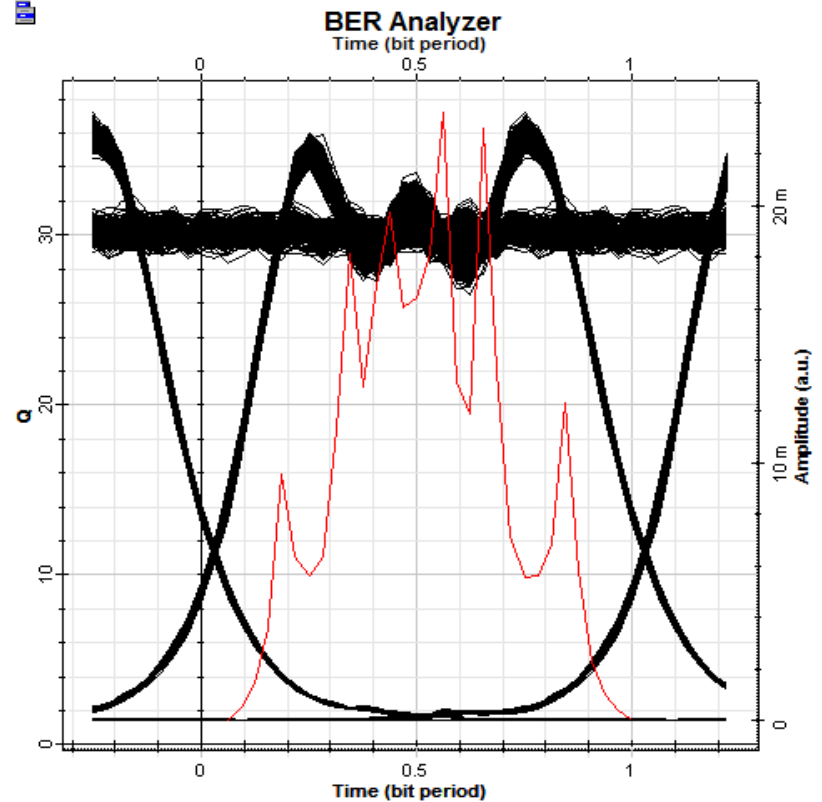

Fig. 8. Q Factor of Low Pass Chebyshev Filter

In this fig.8. eye diagram taken at $1310 \mathrm{~nm}$ frequency of optical source at $10 \mathrm{~mW}$ input power low pass chebyshev filter value of $\mathrm{Q}$ factor is 37.24 came after simulation in optisystem software.

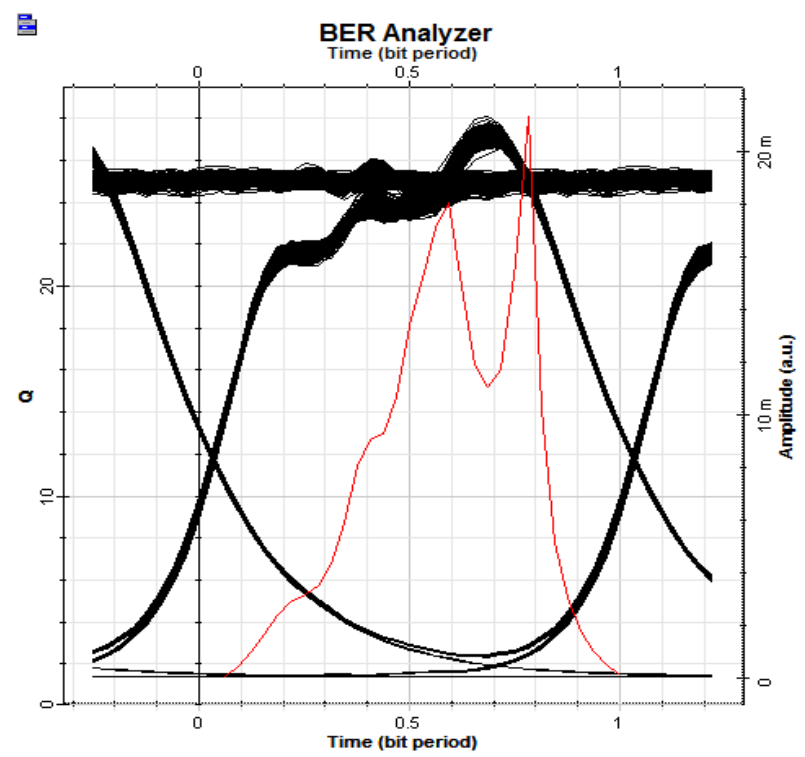

Fig. 9. Q Factor of Low Pass Butterworth Filter

In this eye diagram taken at $1310 \mathrm{~nm}$ frequeny of optical source at $10 \mathrm{~mW}$ input power $\mathrm{Q}$ factor for low pass butterwoth filter came 28.10 .

\section{1 BIT ERROR RATE SIMULATION}

BER rate response taken on $1550 \mathrm{~nm}$ frequency of optical source at $10 \mathrm{~mW}$ power. Bessel filter provides constant group delay due to this eye opening height is more. Bit error rate of Bessel filter is minimum.

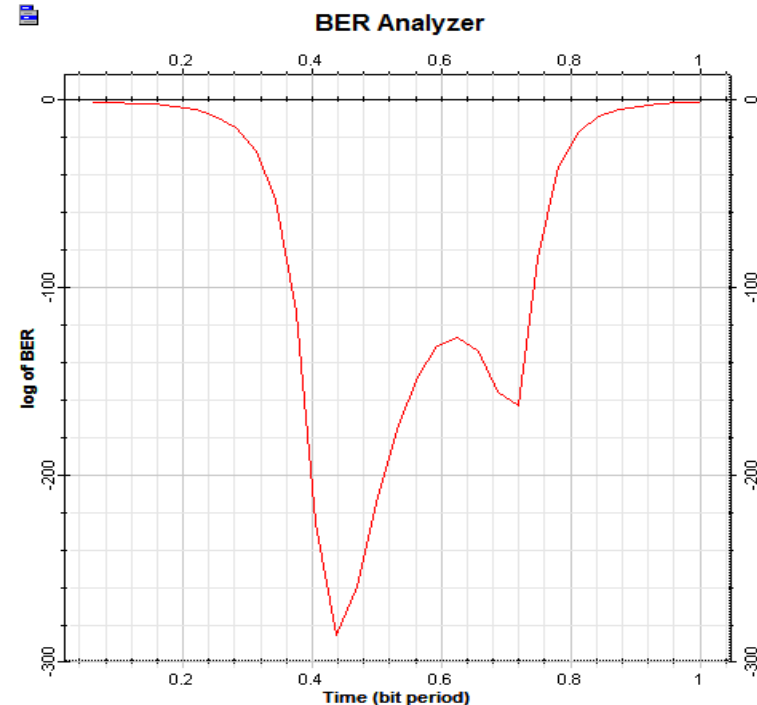

Fig. 10. BER curve of Bessel Filter Min. BER=3.303716e286

In Fig. 10. shows that Bessel filter has minimum bit error rate at $1550 \mathrm{~nm}$ frequency at $10 \mathrm{~mW}$ power.

Table 2: BER ANAYLSIS OF DIFFERENT FILTERS

\begin{tabular}{|l|l|l|}
\hline Filter Type & Eye Height & Min.BER \\
\hline Chebyshev & 0.016 & $1.4125 \mathrm{e}-096$ \\
\hline Butterworth & 0.0158 & $5.1976 \mathrm{e}-124$ \\
\hline Bessel & 0.0173 & $3.303716 \mathrm{e}-286$ \\
\hline
\end{tabular}

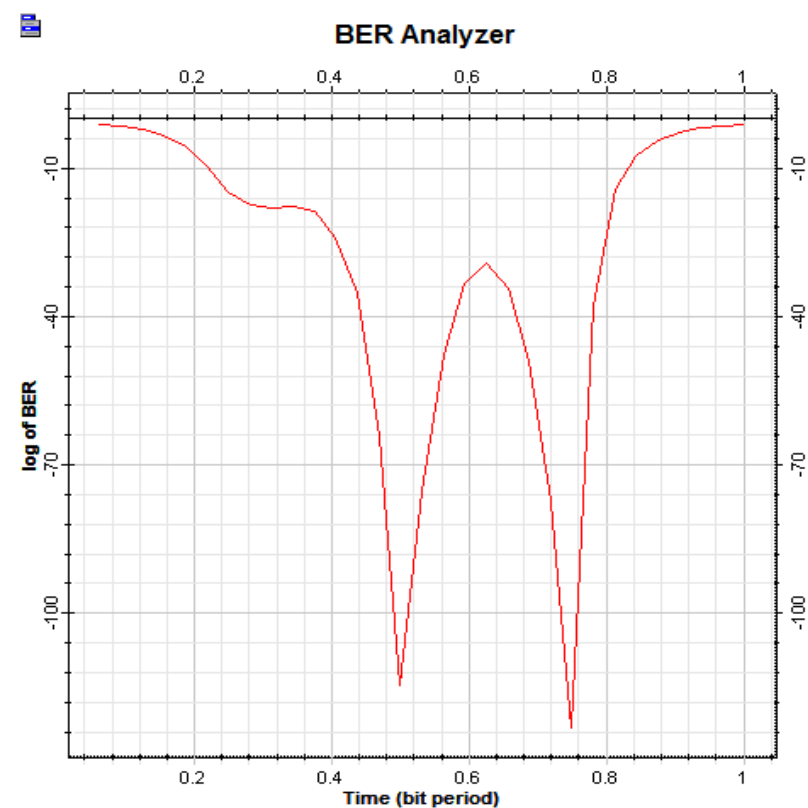

Fig. 11. BER curve of Butterworth filter Min. BER=5.1976e124 
軍

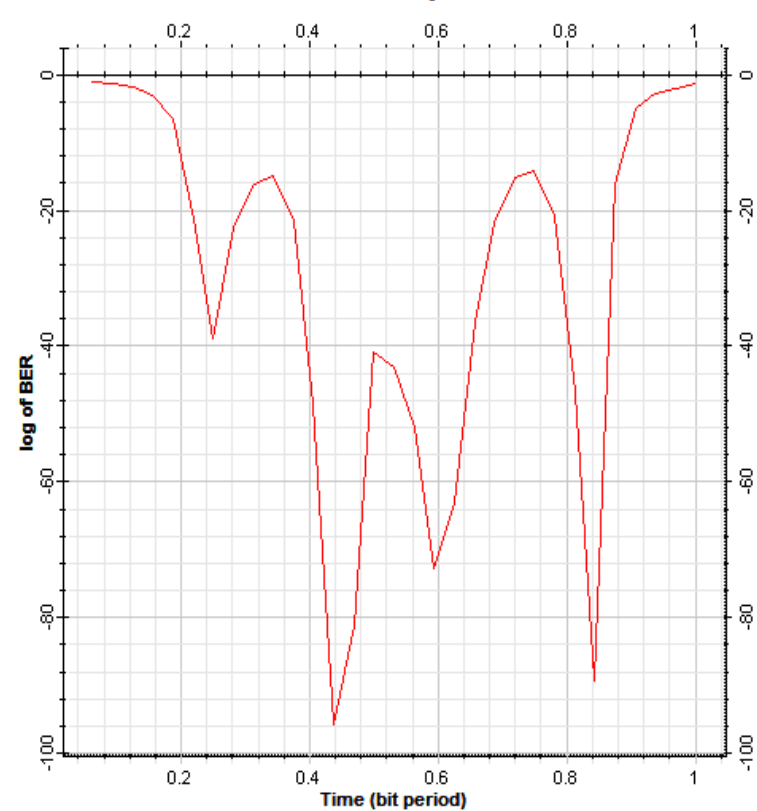

Fig. 12. BER curve of Chebyshev Min. BER=1.4125e-096

At $1310 \mathrm{~nm}$ laser power chebyshev filter has BER 6.3453e-304 and butterworth filter bit error rate is 7.94125e-176. But Bessel filter has minimum BER.

\section{CONCLUSION}

It is concluded that APD photodiode gives a better Performance in given optical system in comparison with PIN photodiode. Low pass Bessel filter shows the best performance among the other filters at $1310 \mathrm{~nm}$ wavelength.

Bessel filter provides constant group delay. Eye opening height is more in Bessel filter. It gives minimum Bit error rate in received signal.

Semiconductor optical amplifier reduces the noise in optical system. It improves the quality of received signal. It provides lower bit error rate upto $20 \mathrm{~km}$ optical fiber.

\section{ACKNOWLEDGMENT}

I would like to acknowledge Assistant Professor Chakresh kumar of USICT, Guru Gobind Singh Indraprastha University his encouragement and cooperation during this project.

\section{REFERENCES}

[1] F.Koyama and H.Uenohara," Noise suppression and optical ASE modulation in saturated semiconductor optical amplifiers," Conference on Signals, Systems and Computers, 7-10 Nov2004, Pacific Grove, CA, USA.

[2] A.V. Poliakov,'Increase of signal-to-noise ratio in fiber-optic systems with avalanche photodiode," proc. of 1 st international conference on advanced optoelectronics and lasers, 16-20 sept. 2003, alushta, crimea, Ukraine.

[3] A.D. Mccoy ,P. Horak, B.C. Thomsen , M. Ibsen And D.J. Richardson," Noise suppression of incoherent light using a gain-saturated SOA implications for spectrum-sliced WDM systems," Journal of Lightwave Technology, 22 August 2005.

[4] y.-y. won ,h.-c. kwon, and s.-k. han," Reduction of optical beat interference using gain-saturated RSOA in upstream $\mathrm{wdm} / \mathrm{scm}$ optical links,"vol no.1, pp. 61 64, april 2007.

[5] A. DEMIR,'Noise analysis for optical fiber communication systems," Int. Conf.international conference on computer aided design, 9-13 Nov. 2003, San Jose, CA, USA, USA.

[6] C.R. Menyuk,"Modeling Noise In Optical Fiber Communications Systems," Optical Fiber Communication Conference, 2004, Los Angeles, CA, USA, USA.

[7] D. Seyringer, F. Uherek, J. Chovan and A. KuzmaDesign," Simulation And Evaluation Of AWG Based Demultiplexers," The Ninth Int.Conf. on Advanced Semiconductor Devices and Mircosystems, 11-15 Nov. 2012,Smolenice,Slovakia. 